\title{
Aprendizagem Colaborativa com Suporte Computacional no Ambiente de Trabalho: Um Mapeamento Sistemático da Literatura
}

\author{
Gildásio da Costa Teixeira ${ }^{1}$, Apuena Vieira Gomes ${ }^{1}$, Itamir de Morais B. Filho ${ }^{1}$ \\ ${ }^{1}$ Instituto Metrópole Digital - Universidade Federal do Rio Grande do Norte (UFRN) \\ Caixa Postal 1524 - 59.078-970 - Natal - RN - Brasil \\ \{gildasio, apuena, itamir.filho\}eimd.ufrn.br
}

\begin{abstract}
Computer supported collaborative learning (CSCL) brings significant contributions to the teaching and learning process, however we envision the use of this pedagogical tool, in addition to the classroom. This work presents the synthesis of the results of a systematic mapping of the literature on the use of CSCL at Work. The main findings are: collaborative applications tend to be more efficient, when in their design, awareness is included; individuals consider it important to have the perception of knowledge created in collaborative activities; it is possible to measure and evaluate collaboration through computational artifacts; that formative feedbacks influence learning and the need for further discussion on the subject due to the scarcity of works published in Portuguese on the subject and the failure to identify techniques for forming CSCL groups with a focus on work environments.
\end{abstract}

Resumo. Aprendizagem Colaborativa com Suporte Computacional (CSCL) traz contribuições significativas no processo de ensino e aprendizagem, contudo vislumbrar-se a utilização desta ferramenta pedagógica, além da sala de aula. Este trabalho apresenta a sintetização dos resultados de um mapeamento sistemático da literatura sobre a utilização da CSCL em ambientes organizacionais. Os principais achados são: aplicações colaborativas tendem a ser mais eficientes, quando em seu design, a conscientização é incluída; indivíduos consideram importante terem a percepção dos conhecimentos criado em atividades colaborativas; é possivel medir e avaliar a colaboração através de artefatos computacionais; que feedbacks formativos influenciam a aprendizagem e a necessidade de maior discussão sobre o assunto devido à escassez de trabalhos publicados em língua portuguesa sobre o tema e a não identificação de técnicas de formação de grupos CSCL com foco em ambientes de trabalho.

\section{Introdução}

Aprendizagem Colaborativa com Suporte Computacional (CSCL) é um processo de ensino e aprendizagem, que aglutina conceitos de algumas áreas do conhecimento (pedagogia, psicologia e computação), e busca através de estratégias de formação de grupos fazer com que possa ser gerada uma cultura de co-criação do conhecimento mediante discussões, argumentações, troca de ideias e resoluções de conflitos. Contribuindo para o desenvolvimento de habilidades individuais e sociais [Duque Reis et al. 2015].

Segundo Dillenbourg e Fischer (2007) a CSCL no ambiente educacional, seja nos diversos níveis de ensino, já demonstrou inúmeras contribuições, porém quando falamos em aprendizagem colaborativa em ambientes organizacionais tais contribuições necessitam ser 
melhor agrupadas, sistematizadas e metodologicamente melhor explicadas para um entendimento explícito de possíveis ganhos em utilizá-las [GOGGINS; JAHNKE, 2010].

Isto posto, para conhecimento do Estado da Arte do assunto abordado em uma pesquisa de mestrado cuja problemática está centrada na ausência de equilíbrio nos níveis de conhecimento entre os membros que compõem a equipe da organização pesquisada, completam a problemática a ausência de sistematização na transferência de conhecimento e a necessidade de compreender como ocorre a aprendizagem nesta equipe, optou-se por um Mapeamento sistemático da literatura (MSL).

Kitchenham e Charters (2007) definem o MSL como sendo um dos tipos de estudos secundários que segue um processo de pesquisa metodologicamente bem definido onde é possível: identificar, analisar, interpretar as evidências disponíveis relacionados com um tópico de pesquisa de maneira não tendenciosa, auditável e repetível.

Este MSL é parte integrante de uma pesquisa de dissertação de um mestrado que propõe a criação de uma metodologia de colaboração, comunicação e aprendizagem para equipes de TI. Sendo assim os achados do MSL contribuíram no percurso metodológico e na indicou trilhas para a resolução do problema de pesquisa.

\section{Método}

O MSL desta pesquisa tem como objetivo principal encontrar e relatar quais ferramentas, cenários e soluções do campo da CSCL que são utilizados em ambientes organizacionais diversos. As fases do protocolo de condução do mapeamento, segundo Petersen et al (2008) são: definição das questões de pesquisa, definição da string de pesquisa, definição dos critérios de inclusão/exclusão, seleção dos estudos relevantes, extração e síntese dos resultados. Foi definido um escopo de busca de 10 anos (2010 a 2019).

\subsection{Definição das questões de pesquisa}

No intuito de cumprir o objetivo do trabalho foram formuladas 03 questões de pesquisa para o mapeamento Sistemático da Literatura:

\section{> Quais são as aplicações, cenários e soluções do campo da CSCL utilizadas em ambientes organizacionais?}

\section{> Como é medida a colaboração da aprendizagem em ambientes de trabalho?}

> Existe alguma técnica específica para formação de grupos CSCL no ambiente de trabalho?

\subsection{Definição String de busca}

Com as questões de pesquisa definidas o processo de mapeamento, iniciou-se com a definição da string de pesquisa a ser utilizada nos motores de busca. Com base em leituras anteriores, o quadro 01 foi montado com os principais termos da área e seus sinônimos e traduções.

Quadro 01 - Termos que deram origem a string de pesquisa.

\begin{tabular}{|l|l|l|}
\hline \multicolumn{1}{|c|}{ Termos } & \multicolumn{1}{|c|}{ Sinônimos } & \multicolumn{1}{c|}{ Tradução } \\
\hline CSCL & $\begin{array}{l}\text { Computer Supported Collaborative } \\
\text { Learning, Computer-Supported } \\
\text { Collaborative Learning }\end{array}$ & $\begin{array}{l}\text { Aprendizagem Colaborativa com suporte } \\
\text { computacional, Aprendizagem } \\
\text { Colaborativa com suporte de tecnologia. }\end{array}$ \\
\hline
\end{tabular}


IX Congresso Brasileiro de Informática na Educação (CBIE 2020)

Anais do XXXI Simpósio Brasileiro de Informática na Educação (SBIE 2020)

\begin{tabular}{|l|l|l|}
\hline Ambiente de trabalho & Ambiente organizacional & Workplace, in service, at work, corporate \\
\hline Formação de grupos & Construção de equipes & $\begin{array}{l}\text { Group formation, peer learning, team } \\
\text { formation }\end{array}$ \\
\hline
\end{tabular}

Fonte: Elaborado pelos autores

Com base nos termos do quadro 01 foram elaboradas duas strings uma em língua inglesa e outra em português. A string em língua inglesa foi aplicada no motor de busca Scopus ${ }^{1}$ que indexa as principais bases bibliográficas: IEEE, ACM digital, Science Direct, Springer, Conferências Internacional CSCL, International Journal of CSCL e a rede social cientifica research gate. A string em português foi aplicada no motor de busca Google Acadêmico. A base bibliográfica da Comissão Especial de Informática na Educação(CEIE) também foi consultada, contudo, optou-se por uma busca manual utilizando termo pôr termo da string, visto que a citada base demonstrou inconsistências nos resultados utilizando a string de busca completa.

Quadro 02: Strings de pesquisa e resultados por bases bibliográficas

\begin{tabular}{|l|l|c|}
\hline \multicolumn{1}{|c|}{ Motor de Pesquisa } & \multicolumn{1}{|c|}{ String } & Resultado \\
\hline Scopus & $\begin{array}{l}\text { "collaborative learning*" OR "peer learning" AND } \\
\text { ("computer sup*" AND "computer-sup*") OR "CSCL at } \\
\text { work" OR "CSCL@work" OR "group formation in } \\
\text { CSCL" OR "corporate" OR "in service" OR "workplace" }\end{array}$ & 1716 \\
\hline Google Acadêmico & $\begin{array}{l}\text { "Aprendizagem Colaborativa" OR "Aprendizagem por } \\
\text { pares" OR “Ambientes colaborativos de aprendizagem" } \\
\text { AND (com suport* OR apoio comp*) OR "Formação de } \\
\text { Grupos" AND "Corporativo" AND "em Serviço" }\end{array}$ & 114 \\
\hline CEIE & Busca manual & 08 \\
\hline
\end{tabular}

Fonte: Elaborado pelos autores

Ao final do processo de busca foram gerados os arquivos bibtex nos motores Scopus e Google Acadêmico e ambos foram organizados em formas de planilha eletrônica (https://bityli.com/FB73r) e nos softwares Zotero e StArt. Os resultados da base CEIE foram incluídos de forma manual em ambas as ferramentas.

Com o término da seleção dos trabalhos foram definidos alguns critérios de inclusão e exclusão para que a seleção dos estudos ocorresse.

\section{Quadro 03 - Critérios de Inclusão/Exclusão dos trabalhos encontrados}

\begin{tabular}{|l|l|}
\hline \multicolumn{1}{|c|}{ Critérios de Inclusão } & \multicolumn{1}{|c|}{ Critérios de Exclusão } \\
\hline $\begin{array}{l}\text { CI01 - Artigos Publicados no período de janeiro de } \\
2010 \text { a dezembro de 2019; }\end{array}$ & $\begin{array}{l}\text { CE01 - Artigos que não atendem os critérios de } \\
\text { inclusão; }\end{array}$ \\
\hline CI02 - Artigos em português, inglês ou espanhol; & CE02 - Artigos duplicados; \\
\hline
\end{tabular}

\footnotetext{
${ }^{1}$ https://www.scopus.com/
} 
CI03 - Artigos que demonstram, propõe e ou relatam, processos, estratégias, boas práticas, experiências, soluções, metodologias, projetos, ferramentas, formação de grupos, métodos, e arquiteturas para utilização de CSCL no ambiente de trabalho;

CI04 - Artigos completos.
CE03 -Artigos onde não foi possível o acesso ao texto completo;

CE04 - O artigo é uma versão anterior de um estudo mais completo;

CE05 - Artigos em que a sigla CSCL não faça referência a Computer-Supported Collaborative Learning.

Fonte: Elaborado pelos autores

\subsection{Seleção dos Estudos relevantes}

A seleção dos estudos relevantes foi realizada em três etapas detalhadas na Figura 01 . Na fase 01, a string de pesquisa foi executada conforme Quadro 01. Ainda na fase 01 foram retirados 22 artigos duplicados, para tal fim a ferramenta StArt foi utilizada.

$\mathrm{Na}$ sequência, na fase 02 , foi feita a leitura de título, resumo e palavras-chave dos 1.816 artigos restantes reduzindo a quantidade de trabalhos para 85 que estão listados na planilha (https://bityli.com/FB73r), e foram lidos por completo e também aplicado os critérios de inclusão e exclusão (Quadro 03). Como resultado da leitura completa dos 85 artigos selecionados, 28 artigos atenderam aos critérios de inclusão definidos e foram encaminhadas para a fase 03 que consiste da extração e síntese dos achados.

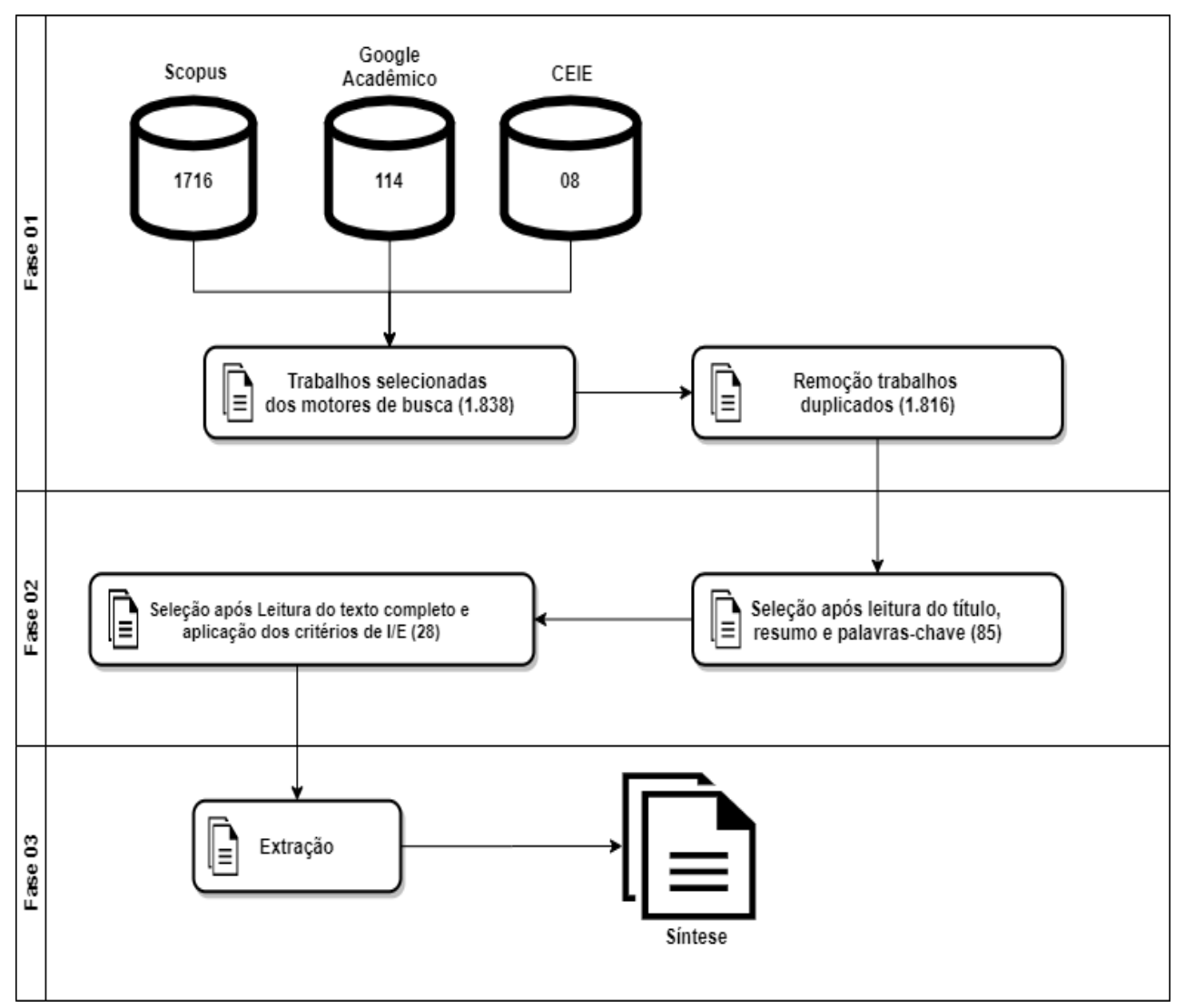

Figura 01 - Processo de seleção de trabalhos relevantes

Fonte: Elaborado pelos autores 
IX Congresso Brasileiro de Informática na Educação (CBIE 2020)

Anais do XXXI Simpósio Brasileiro de Informática na Educação (SBIE 2020)

\section{Extração de dados}

Com os 28 trabalhos selecionados foi possível verificar que em relação ao tipo de publicação $53,6 \%$ são de periódicos, $32,1 \%$ são de conferências e $14,3 \%$ foram publicados em forma de capítulo de livro.

Com base nos resultados dos motores de busca algumas bases bibliográficas foram consultadas para obtenção do trabalho em sua forma completa. Os resultados foram os seguintes: Springer link (15 trabalhos), Research gate (08), IEEE (05) as bases Science Direct, ACM Digital, Academia Edu, Renote e Rbie forneceram 01(um) trabalho cada.

\section{1 Síntese dos Resultados}

Com a seleção final de 28 artigos, foi realizada uma análise para aferir os dados com os as questões de pesquisa levantadas no início do processo do mapeamento Sistemático da Literatura. A discussão sobre os achados é descrita a seguir.

\subsection{1 (QP01) Quais são as aplicações, cenários e soluções do campo da CSCL utilizadas em ambientes organizacionais?}

Em relação às aplicações, cenários e soluções da aprendizagem colaborativa com suporte computacional em ambiente organizacional o trabalho de Collazos et al.(2019) traz uma teoria descritiva, para a implementação de conscientização, no desenvolvimento de ferramentas colaborativas. Os autores reforçam que soluções colaborativas centradas em conscientização tem demonstrado mais eficácia no processo colaborativo, e traz como vantagem a melhoria nas relações sociais e consequentemente promove um sentimento de bem estar entre os participantes.

O modelo de Collazos et al. (2019) é composto por 5 (cinco) fases: Metas de conscientização; identificação de informações de conscientização; Modelagem para integração da conscientização em softwares desenvolvidos por meio MDD (Model-Driven Development); distribuição; conscientização nas interfaces dos usuários. O modelo é capaz de organizar e classificar, termos, aspectos e definições ajudando assim na construção de ferramentas colaborativas.

O modelo de colaboração de Murphy (MURPHY, 2004) busca levar colaboração para ambientes virtuais de aprendizagem (AVA) através da implementação de 06 fases: presença social, articulação das perspectivas individuais, reflexão sobre as perspectivas alheias, construção compartilhada de perspectivas e significados, compartilhamento da construção de objetivos e produção compartilhada de artefatos.

Schaefer et al. (2019), demonstra a aplicação do modelo de Murphy ampliado. O lócus da pesquisa é uma repartição pública, as pesquisadoras partem da premissa que as análises das discussões assíncronas potencializam o envolvimento cognitivo que a aprendizagem colaborativa requer, que a presença social é um pré-requisito dinâmico para a aprendizagem colaborativa e aumenta o aprendizado, como também a facilita o trabalho entre pares. Foram analisados 1170 comentários que surgiram em um curso online entre os colaboradores da repartição, concluindo que a presença social quando adicionada na aprendizagem colaborativa traz consigo aumento da reflexão e da co-construção do conhecimento.

O impacto dos fatores intrínsecos na aprendizagem colaborativa em AVA é demonstrado por Singh [2019]. O estudo foca na capacidade de autopercepção de criação de 
conhecimento de quando se trabalha de forma colaborativa. Também são discutidos no trabalho conceitos como: criação de conhecimento, nível de confiança entre pares, influência entre pares, expectativa em resultados pessoais.

Cheng, Li e Zhao (2015), demonstram como um sistema CSS (Collaborative Support System), executado em WAMP, pode contribuir para alguns aspectos impeditivos de uma colaboração eficaz. Os autores tratam da baixa participação dos indivíduos, distração e ineficiência. Um estudo de caso aplicado com 33 especialistas de variados segmentos, sendo conduzido com os instrutores de cada grupo treinados no CSS, logo após cada instrutor em sua organização buscou utilizar a ferramenta para o estímulo da discussão e avaliação de problemas de várias perspectivas, reconstrução e co-construção do conhecimento. Após a resolução das atividades colaborativas cada instrutor respondeu um questionário e foi realizado uma entrevista. Os participantes apontaram os seguintes vantagens em utilizar o sistema para implementação da colaboração: facilitação por um líder nas atividades colaborativas, aumento de participação(incluindo pessoas introvertidas) fazendo com que o grande número de interações otimize a criação do conhecimento e o aumento da concentração.

Uma importante contribuição para o campo de estudos da CSCL no ambiente de trabalho é apresentado por Goggins e Jahnke (2010b) através de uma meta análise de 08 (oito) trabalhos na área os autores desenvolveram as seguintes teses de design: O aprendizado ocorre em locais inesperados e incomuns, especialmente por meio das mídias sociais; as atividades de aprendizagem incorporam feedback de diversas pessoas, que não estão disponíveis dentro dos limites organizacionais tradicionais; a aprendizagem deve ser visível através dos limites estabelecidos. Um dos objetivos de trazer a CSCL para o ambiente de trabalho é a possibilidade da junção do conhecimento já existente com a possibilidade da coconstrução do conhecimento através das interações do grupo, sendo essa a principal características que distingue o aprendizado colaborativo do aprendizado individual e organizacional. $\mathrm{O}$ trabalho traz um modelo de design geral para aplicação da aprendizagem colaborativa no trabalho.

No trabalho de Hartswood et al. (2013) é relatado um estudo de caso em um serviço médico de imagens(mamografia). O trabalho objetiva que através da CSCL os treinamentos que ocorrem no local de trabalho possam ajudar no desenvolvimento dos profissionais, melhorar a interação e colaboração entre instrutores e estagiários. Os pesquisadores focam em colocar em prática o que eles chamam de "tecnologias de participação" e de como os recursos da aprendizagem colaborativa podem coexistir num ambiente onde os processos de trabalho, geralmente são rígidos e pouco flexíveis.

Um modelo de comunicação baseado em três funções (comunicador, destinatário e facilitador) é apresentado por [KIENLE 2013]. São demonstrados 2 (dois) estudos de caso sendo um com a comunicação assíncrona e outro mostrando a facilitação da comunicação síncrona. Conclui-se que a facilitação na comunicação síncrona tem uma tendência de acontecer de forma satisfatória quando o facilitador utiliza palavras instrutivas em suas contribuições ao grupo. Contudo, a citada facilitação não foi suficiente para o término com êxito de atividades de colaboração mais complexas, para esses casos é necessário o facilitador apresente grande parte da solução de forma concreta para que o grupo tenha condições de completar a tarefa. Destarte na comunicação assíncrona a estratégia de comunicação foi alcançada.

Distribuição da cognição, aprendizado sob demanda, estruturação e soluções 
integradas de problemas, comunidades de interesse, metadesign, culturas de participação e criatividade social são demonstradas na estrutura conceitual de [Fischer 2013]. O autor defende que a CSCL traz vantagens tanto para os indivíduos como para as organizações e traz conceito de "aprender quando a resposta não é conhecida". Como impacto, a CSCL no trabalho tem a ênfase de trazer opções para a aprendizagem fora do ambiente escolar e mudar o paradigma que em ambientes organizacionais o ensino só ocorre em forma de treinamentos, contribuindo assim com a educação de adultos(andragogia).

Koops et al. (2011) explora como as discussões assíncronas do CSCL pode agregar valor à aprendizagem colaborativa num ambiente de ensino hospitalar com alunos que cursam o internato para conclusão do curso de medicina. $O$ estudo foi conduzido partindo de um problema clínico onde subgrupos foram criados e os participantes discorreram sobre preparação, participação, design e ganhos de conhecimento. Conclui-se que a CSCL através dos seus feedbacks formativos influenciou positivamente no aprendizado.

\subsection{2 (QP02) Como é medida a colaboração da aprendizagem em ambientes de trabalho?}

Em relação às principais formas de verificar, avaliar e medir a colaboração quando se utiliza a CSCL no ambiente de trabalhos os autores classificaram os achados em duas categorias (conceitual e prático). As soluções conceituais encontradas foram: taxionomia, modelo de colaboração, observação(instrutor e ambiente), k.nowledge creation, conjunto de aprendizado, modelo conceitual próprio, teoria fundamentada, métodos mistos. Já em relação às soluções práticas os achados mostram uma solução CSS e outra web. Os apanhados demonstram a predominância de modelos conceituais para medição da colaboração.

Um dos grandes desafios encontrados nas pesquisas CSCL é como medir colaboração e suas interações. Ishikawa et al. (2018) lembra a importância no controle das interações entre os participantes de atividade colaborativas. Stahl (2006) mostra que entre outros conceitos de CSCL que a medição da colaboração pode ser feita através de artefatos computacionais, o autor reconhece as contribuições das pesquisas quantitativas em para tal fim, contudo lembra que nem toda as atividades colaborativas podem ser medidas desta forma.

\subsection{3 (QP03) Existe alguma técnica específica para formação de grupos CSCL no ambiente de trabalho?}

Em relação a formação de grupos, 53\% dos trabalhos selecionados são referentes a temática. Sendo que 04 trabalhos são trabalhos secundários (RSL e Mapeamento). Os dados coletados dos trabalhos mostram que existem as seguintes estratégias para formação de grupos: algoritmos diversos, afetividade, Modelo Multi-Agente Fuzzy (lógica difusa), aprendizagem de máquina, estilos de aprendizagem, satisfação (características de aprendizagem, papéis na equipe e networking), Software para implementação de grupo através do auto relato do indivíduo.

A figura 02 demonstra que a estratégia predominante e a utilização de algoritmos para automatização e customização na tarefa de formação de grupos sejam homogêneas e ou heterogêneos. 


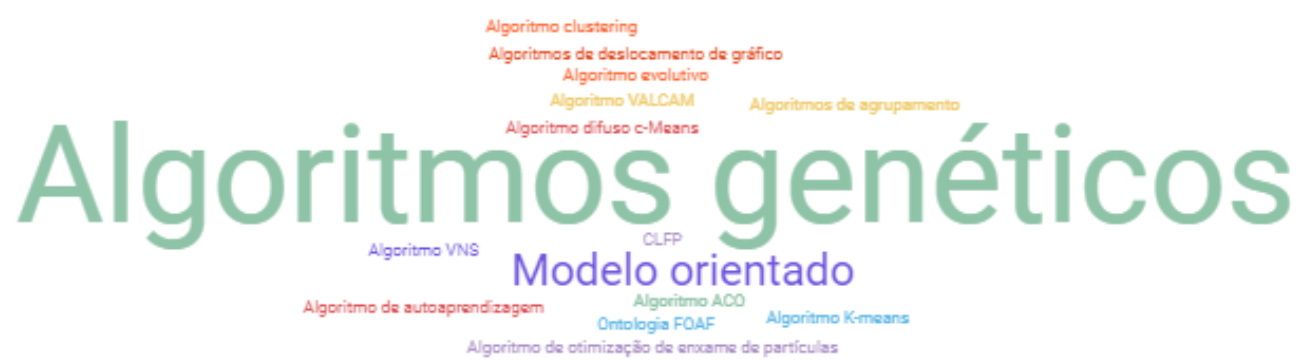

Figura 02 - Nuvem de palavras algoritmos utilizados para formação grupos CSCL. Fonte: elaborado pelos autores

Embora não tenha sido encontrado uma técnica específica de formação de grupos para CSCL no ambiente de trabalho, para a realidade do grupo de participantes (13) objeto da pesquisa de mestrado, os trabalhos de Duque e Reis et. al (2015), Balmaceda et. al (2014), Mehennaoui et al. (2014) e Costa Quarto et al. (2016). Tendem a oferecer a solução de agrupamento mais eficaz.

\subsection{Ameaças à Validade}

A principal limitação deste mapeamento sistemático está no fato que pode ocorrer equívocos na seleção de publicações e na extração de dados. Mesmo seguindo rigorosamente o protocolo definido, como descrito na seção 2.3 (Figura 01), que visa garantir que o processo de seleção seja imparcial. Outra limitação é a string de pesquisa usada, descrita na seção 2.2, a despeito de ter sido definida com base nas questões da pesquisa e termos recorrentes da área, existe o risco de alguns estudos serem omitidos.

Com relação às bases de bibliográficas e motores de busca, a limitação está no uso da Scopus, que embora indexe bases diversas, a inclusão no Scopus, uma vez publicado um artigo, leva algum tempo e, portanto, existe o risco de alguns estudos já publicados ainda não terem sido incluídos como por exemplo trabalhos publicados no último trimestre de 2019.

\section{Considerações Finais}

Este trabalho apresentou um Mapeamento Sistemático da Literatura quem tem o intuito de apresentar o Estado da Arte na área CSCL com foco em suas aplicações, ferramentas, cenários, conceitos e soluções em ambientes organizacionais.

Os principais resultados obtidos demonstram que ao projetar aplicações colaborativas a conscientização(sensação de pertencimento) é um fator importante para se ter nos requisitos da aplicação, que tão importante quanto a criação do conhecimento na colaboração é a autopercepção dos indivíduos quanto a isto. Também foi extraído que artefatos computacionais são capazes de medir e avaliar colaboração e interação e que umas das aplicações encontradas foi capaz de prover aumento de participação (entre pessoas introvertidas) e que graças a isso o número o número de interações aumentou. Em outra pesquisa foi encontrado um modelo conceitual e generalista para a utilização da CSCL em serviço,

Em relação a comunicação na CSCL uma pesquisa demonstrou que que feedbacks formativos influenciam positivamente no aprendizado. Que a medição da colaboração em ambientes organizacionais é majoritariamente conceitual dispondo de um número reduzidos de soluções práticas. Em relação a formação de grupos não foi encontrado uma técnica específica para CSCL at work, contudo foi possível identificar 04 técnicas que podem balizar 
IX Congresso Brasileiro de Informática na Educação (CBIE 2020)

Anais do XXXI Simpósio Brasileiro de Informática na Educação (SBIE 2020)

a formação de grupo na pesquisa de mestrado que deu origem a este MSL. Também se destaca o fato de que não foram encontrados trabalhos publicados em português que demonstrasse algum cenário da utilização da CSCL no ambiente de trabalho.

Destarte, o MSL demonstra sua contribuição na apresentação do estado da arte da CSCL no trabalho e como parte importante da composição de uma pesquisa de mestrado e servindo como ponto de partida para trabalhos futuros semelhantes.

\section{Referências}

BALMACEDA, J. M.; SCHIAFFINO, S.; ANDRÉS DÍAZ-PACE, J. Using constraint satisfaction to aid group formation in CSCL. Inteligencia Artificial, v. 17, n. 53 SPEC. ISS., p. 35-45, 2014.

CHENG, X.; LI, Y.; ZHAO, Y. Can we solve low participation, distraction, and inefficiency? A case study of distributed collaborative learning in industries. (B. T. X. Sprague R.H., Ed.)Proceedings of the Annual Hawaii International Conference on System Sciences. Anais...IEEE Computer Society, 2015.

COSTA QUARTO, C. et al. SAMAR: Um Sistema Autônomo de Mensuração de Auto Relatos no auxílio à Formação de Grupos para o campo CSCL. RENOTE, v. 13, n. 2, 7 jan. 2016.

COLLAZOS, C. A. et al. Descriptive theory of awareness for groupware development. Journal of Ambient Intelligence and Humanized Computing, v. 10, n. 12, p. 4789-4818, 2019.

DILLENBOURG, P.; FISCHER, F. Computer-supported collaborative learning: The Basics. p. 22, 2007.

DUQUE REIS, R. C. et al. Estado da Arte sobre Afetividade na Formação de Grupos em Ambientes Colaborativos de Aprendizagem. Revista Brasileira de Informática na Educação, v. 23, n. 03, p. 113, 29 dez. 2015.

FISCHER, G. A Conceptual Framework for Computer-Supported Collaborative Learning at Work. In: GOGGINS, S. P.; JAHNKE, I.; WULF, V. (Eds.). . Computer-Supported Collaborative Learning at the Workplace: CSCL@Work. Boston, MA: Springer US, 2013. p. 23-42.

GOGGINS, S. P.; JAHNKE, I. Computer supported collaborative learning at work: CSCL at work. Proceedings of the 16th ACM International Conference on Supporting Group Work, GROUP'10. Anais...Sanibel Island, FL: 2010.

HARTSWOOD, M. et al. Technologies of Participation: A Case Study of CSCL@Work in Mammography. In: GOGGINS, S. P.; JAHNKE, I.; WULF, V. (Eds.). Computer-Supported Collaborative Learning at the Workplace: CSCL@Work. Boston, MA: Springer US, 2013. p. 113138.

ISHIKAWA, E. C. M. et al. Contribuições de um Objeto de Aprendizagem Colaborativa no Contexto da CSCL. RENOTE-Revista Novas Tecnologias na Educação, v. 16, n. 2, p. 61-70, 2018.

KIENLE, A. The Role of Communication and Facilitation for CSCL@Work. In: GOGGINS, S. P.; JAHNKE, I.; WULF, V. (Eds.). . Computer-Supported Collaborative Learning at the Workplace: CSCL@Work. Boston, MA: Springer US, 2013.p. 185-202.

KITCHENHAM, B.; CHARTERS, S. Guidelines for performing Systematic Literature Reviews in Software Engineering. 2007. 
IX Congresso Brasileiro de Informática na Educação (CBIE 2020)

Anais do XXXI Simpósio Brasileiro de Informática na Educação (SBIE 2020)

KOOPS, W. et al. Computer-supported collaborative learning in the medical workplace: Students' experiences on formative peer feedback of a critical appraisal of a topic paper. Medical Teacher, v. 33, n. 6, p. e318-e323, 2011.

MEHENNAOUI, Z. et al. A new approach for grouping learners in CSCL systems. International Conference on Multimedia Computing and Systems -Proceedings. Anais...IEEE Computer Society, 2014.

MURPHY, E. Recognising and promoting collaboration in an online asynchronous discussion. British Journal of Educational Technology, v. 35, n. 4, p. 421-431, 2004.

PETERSEN, K. et al. Systematic Mapping Studies in Software Engineering. . In: 12TH INTERNATIONAL CONFERENCE ON EVALUATION AND ASSESSMENT IN SOFTWARE ENGINEERING (EASE). 1 jun. 2008 Disponível em: $<$ https://scienceopen.com/document?vid=6d552894-2cc3-4e2b-a483-41fa48a37ef8>. Acesso em: 9 maio. 2020.

SCHAEFER, T. et al. Fostering online learning at the workplace: A scheme to identify and analyse collaboration processes in asynchronous discussions. British Journal of Educational Technology, v. 50, n. 3, p. 1354-1367, 2019.

SINGH, R. Impact of Intrinsic Motivators on Knowledge Sharing in Virtual Environments: Implications for Workplace Information Literacy and Collaborative Practices. Communications in Computer and Information Science, v. 989, p. 211-220, 2019. 